\title{
Diarreia no paciente crítico: conhecimento e conduta de profissionais de unidades de terapia intensiva
}

\author{
Diarrhea in the critical patient: knowledge \\ and conduct of professionals in intensive \\ care units
}

\section{Beatriz Silva de Sousa Siqueira' ${ }^{1}$ Fernanda Godoi Melo² (1)}

\begin{abstract}
1Autora para correspondência. Faculdade de Medicina da Universidade Federal de Uberlândia (Uberlândia). Minas Gerais, Brasil. bzsouza@yahoo.com.br
\end{abstract} ${ }^{2}$ Hospital de Clínicas da Universidade Federal de Uberlândia (Uberlândia). Minas Gerais, Brasil. ferngmelo@gmail.com

\begin{abstract}
RESUMO | OBJETIVO: Avaliar o conhecimento e conduta de profissionais de saúde em relação à diarreia em pacientes recebendo nutrição enteral. MÉTODOS: Estudo transversal realizado com profissionais de saúde de duas Unidades de Terapia Intensiva de adultos. Os dados foram coletados por meio de um questionário autoaplicável. RESULTADOS: Participaram do estudo 37 profissionais, sendo 17 (45,9\%) auxiliares ou técnicos em enfermagem, $8(21,6 \%)$ enfermeiros, $8(21,6 \%)$ médicos, $3(8,1 \%)$ nutricionistas e $1(2,8 \%)$ profissão omissa. A definição mais comum encontrada para diarreia foi "três ou mais episódios de evacuações líquidas ou semilíquidas/dia." As principais causas citadas para diarreia foram a "infecção" e a "dieta". A atitude/conduta mais observada foi "comunicação à equipe". A maioria (16; 43,2\%) presencia, com muita frequência, dietas interrompidas por causa de diarreia e a "adequação da dieta" e a "capacitação da equipe multiprofissional" foram as soluções mais citadas para se prevenir essa interrupção. A maioria (20; 54\%) dos profissionais não participou de nenhum treinamento sobre diarreias, e 33 profissionais (cerca de 90\%) considera importante a implementação de um protocolo. CONCLUSÃO: Há divergências entre os profissionais de saúde, tanto em seus conhecimentos quanto nas condutas frente ao paciente com diarreia na UTI, o que pode influenciar diretamente no cuidado com o mesmo. A capacitação da equipe multiprofissional e a criação de um protocolo são essenciais.
\end{abstract}

DESCRITORES: Diarreia. Nutrição Enteral. Profissional da Saúde. Cuidados Intensivos. Unidade de Terapia Intensiva.

\begin{abstract}
OBJECTIVE: To evaluate the knowledge and conduct of health care professionals regarding diarrhea in patients receiving enteral nutrition. METHODS: Crosssectional study conducted with health care professionals from two adult Intensive Care Units. Data were collected using a self-administered questionnaire. RESULTS: 37 professionals participated in the study, being 17 (45.9\%) nursing assistants or technicians, $8(21.6 \%)$ nurses, $8(21.6 \%)$ physicians, 3 (8.1\%) nutritionists, and 1 (2.8\%) omitted profession. The most common definition found for diarrhea was "3 or more liquid or semi-liquid stools/day." The main causes cited for diarrhea were "infection" and "diet." The most commonly observed attitude/conduct was "reporting to the team." The majority $(16 ; 43.2 \%)$ witness diets interrupted very often because of diarrhea and "diet adjustment" and "training of the multiprofessional team" were the most cited solutions to prevent this interruption. The majority $(20 ; 54 \%)$ of the professionals did not participate in any training on diarrhea, and 33 professionals (about 90\%) consider the implementation of a protocol important. CONCLUSION: there are divergences among health professionals, both in their knowledge and conducts when dealing with patients with diarrhea in the Intensive Care Unit (ICU), which can directly influence care. The training of the multiprofessional team and the creation of a protocol are essential.
\end{abstract}

DESCRIPTORS: Diarrhea. Enteral Nutrition. Health Professional. Intensive Care. Intensive Care Unit. 


\section{Introdução}

Os pacientes em estado crítico possuem risco imediato de óbito ou de perda de alguma(s) função(ões) orgânica(s), devido a um trauma ou situações clínicas específicas ${ }^{1}$, como nos casos de grandes queimaduras, cirurgias de grande porte, pancreatite aguda, entre outros. Esses pacientes apresentam risco elevado de desnutrição devido fatores como jejum prolongado e hipermetabolismo. A ocorrência de desnutrição em pacientes internados em Unidade de Terapia Intensiva (UTI) é identificada entre 38 a $70 \%$ dos pacientes $2^{2}$. Para atenuar a presença da desnutrição, um dos recursos utilizados é a Terapia Nutricional Enteral (TNE), que diminui o risco de mortalidade e infecções, principalmente quando a terapia é iniciada precocemente, entre as primeiras $24-48$ horas de internação ${ }^{3}$.

A TNE é indicada para pacientes impossibilitados de suprir suas necessidades nutricionais pela alimentação via oral e, apesar de possuir vantagens em relação à nutrição parenteral, está relacionada a algumas complicações mecânicas, gastrointestinais e metabólicas ${ }^{2}$. De acordo com estes autores, complicações gastrointestinais, como a diminuição dos ruídos intestinais, o retardo no esvaziamento gástrico e a diarreia podem acometer até $50 \%$ dos pacientes em UTI dependentes de ventilação mecânica.

Menos da metade dos pacientes internados em UTI recebem o total de suas necessidades nutricionais ${ }^{4}$. A infusão da dieta é frequentemente interrompida para realização de procedimentos, exames, manutenção ou necessidade de reposicionamento dos dispositivos de nutrição enteral, por instabilidade hemodinâmica, procedimentos fisioterápicos e de enfermagem, intolerâncias gastrointestinais e práticas errôneas da equipe ${ }^{5}$, o que faz com que os pacientes recebam, em média, apenas $80 \%$ do que é prescrito 4 . A interrupção ocorre em aproximadamente $85 \%$ dos pacientes e em média de 8 a $20 \%$ do tempo de infusão ${ }^{6}$.

A diarreia é uma complicação comum na UTI, atingindo entre 2 a 95\% dos pacientes, dependendo dos critérios e definições utilizados ${ }^{7}$. A definição de diarreia é diferente de acordo com a literatura, podendo ser definida como a eliminação de fezes líquidas/não forma- das 3 ou mais vezes ao dia; mais que $300 \mathrm{ml}$ de fezes diárias por dia ${ }^{8-9}$ ou a eliminação de fezes moles ou líquidas mais que 3 vezes em um período de 24 horas $^{10}$.

A etiologia da diarreia é multifatorial e fatores diretamente associados à nutrição enteral podem estar envolvidos, como a quantidade de fibras e gorduras, presença de Fermentable Oligosaccharides, Disaccharides, Monosaccharides And Polyols (FODMAPS), densidade calórica, osmolaridade, temperatura, velocidade de infusão e contaminação da dieta enteral. Outras condições também relacionadas são o uso de medicamentos, incluindo os antibióticos (principais contribuintes), a presença de infecções, incluindo infecção por Clostridium difficile, fatores fisiológicos relacionados ao estresse ou doença crítica e condições intrínsecas ao paciente, como hipoalbuminemia 23,7,9. A diarreia pode trazer complicações graves como desnutrição, perda de eletrólitos e desidratação, o que aumenta o tempo de permanência hospitalar e contribui para maior morbimortalidade e custos hospitalares ${ }^{11}$.

No tratamento da diarreia, a dieta enteral não deve ser considerada como causa principal. É necessária a verificação do fator desencadeante a fim de se tomar medidas adequadas, não sendo recomendada a interrupção da dieta ${ }^{2,3,1}$. O manejo correto garante assistência adequada ao paciente, livrando-o dos riscos relacionados ao recebimento inadequado de nutrientes ${ }^{12}$. Sendo assim, é extremamente importante que profissionais de saúde tenham conhecimento a respeito dos fatores que possam desencadear quadros de diarreia em pacientes hospitalizados, para que possam tomar a conduta adequada. Dessa forma, o objetivo do presente estudo foi avaliar o conhecimento e conduta de profissionais de saúde em relação à diarreia em pacientes recebendo nutrição enteral.

\section{Métodos}

Trata-se de um estudo transversal realizado com enfermeiros, técnicos e auxiliares de enfermagem, médicos e nutricionistas, que trabalham em duas Unidades de Terapia Intensiva (UTI) adulto de um hospital universitário. 
A coleta de dados foi realizada no período de novembro a dezembro de 2017. Todos os profissionais encontrados em seus horários de trabalho foram convidados a participar do estudo, sendo explicado aos mesmos os objetivos e procedimentos. Foram incluídos aqueles que aceitaram participar após ler, concordar e assinar o Termo de Consentimento Livre e Esclarecido (TCLE). Foram excluídos aqueles que aceitaram, mas não entregaram o questionário no momento determinado.

O número total de profissionais que trabalham nas UTIs (auxiliares ou técnicos em enfermagem, enfermeiros, médicos e nutricionistas) é de 117 . No momento da coleta foi encontrado e convidado a participar da pesquisa o total de $78(66,7 \%)$ profissionais. Desses, 2 (2,6\%) não quiseram participar e 39 (50,0\%) não entregaram o questionário no tempo determinado, sendo excluídos. A amostra final foi composta de 37 profissionais.

A coleta de dados foi realizada por meio de um questionário autoaplicável, elaborado pelos pesquisadores e composto por 11 itens, incluindo questões discursivas e de múltipla escolha. As questões discursivas incluíram os seguintes aspectos: i) definição de diarreia; ii) conduta/atitude ao se deparar com um paciente com diarreia; iii) registro detalhado de casos de diarreia; iv) causas da diarreia; v) motivos pelos quais a dieta possa causar diarreia; e vi) o que deve ser feito para se evitar a interrupção da dieta em casos de diarreia. Já as questões de múltipla escolha abordaram os itens: i) treinamentos sobre diarreia; ii) a importância dos aspectos a serem observados no paciente com diarreia; iii) a forma como o registro de casos de diarreia é realizado; iv) qual a frequência em que se observa dietas interrompidas por causa de diarreia na prática clínica; e v) se conhece e acha importante a implementação de um protocolo.

Nas questões de múltipla escolha, os profissionais puderam marcar apenas uma alternativa como resposta. No caso das questões discursivas, as respostas semelhantes foram agrupadas em uma determinada classificação. Dessa forma, surgiram classificações que foram apresentadas de acordo com a frequência de sua ocorrência. A análise dos dados foi realizada por meio do software Statistical Package for Social Sciences - SPSS Statistic 23 para determinação de número absoluto e frequência.

O projeto foi aprovado pelo Comitê de Ética em Pesquisa da Universidade Federal de Uberlândia sob o número 2.372.489/2017.

\section{Resultados}

Participaram do estudo 37 profissionais, sendo 17 $(45,9 \%)$ auxiliares ou técnicos em enfermagem, 8 $(21,6 \%)$ enfermeiros, $8(21,6 \%)$ médicos, $3(8,1 \%)$ nutricionistas e $1(2,8 \%)$ profissão omissa. As características sociodemográficas dos participantes encontram-se na tabela 1.

Tabela 1. Características sociodemográficas dos profissionais - Uberlândia, MG - 2017

\begin{tabular}{|c|c|c|c|c|}
\hline Variáveis & $\begin{array}{c}\text { Aux/Técnico } \\
\text { enfermagem } \\
n(\%)\end{array}$ & $\begin{array}{c}\text { Enfermeiro } \\
\text { n (\%) }\end{array}$ & $\begin{array}{l}\text { Médico } \\
\text { n (\%) }\end{array}$ & $\begin{array}{c}\text { Nutricionista } \\
\text { n (\%) }\end{array}$ \\
\hline \multicolumn{5}{|l|}{ Sexo: } \\
\hline Feminino & $14(82,3)$ & $5(62,5)$ & $6(75,0)$ & $3(100,0)$ \\
\hline Masculino & $3(17,6)$ & $3(37,5)$ & $2(25,0)$ & - \\
\hline \multicolumn{5}{|l|}{ Idade (anos): } \\
\hline $20-30$ & $4(23,5)$ & $5(62,5)$ & - & $1(33,3)$ \\
\hline $30-40$ & $7(41,2)$ & $2(25,0)$ & $7(87,5)$ & $2(66,7)$ \\
\hline$>40$ & $3(17,6)$ & $1(12,5)$ & $1(12,5)$ & - \\
\hline \multicolumn{5}{|c|}{ Tempo de trabalho em UTI (anos) } \\
\hline$<1$ & $1(5,9)$ & $1(12,5$ & - & - \\
\hline $1-3$ & $9(52,9)$ & $3(37,5)$ & $1(12,5)$ & $1(33,3)$ \\
\hline$>3-6$ & $4(23,5)$ & $2(25,0)$ & $6(75,0)$ & - \\
\hline$>6$ & $2(11,8)$ & $2(25,0)$ & $1(12,5)$ & $2(66,7)$ \\
\hline
\end{tabular}


As respostas obtidas pelos profissionais quando questionados sobre a definição de diarreia foram heterogêneas, podendo observar 16 distintas. A maioria dos profissionais $(11 ; 29,7 \%)$, considera como a definição de diarreia "três ou mais episódios de evacuações líquidas ou semilíquidas/dia)", como visto no gráfico 1.

Gráfico 1. Respostas encontradas pelos profissionais sobre a definição de diarreia - Uberlândia, MG - 2017

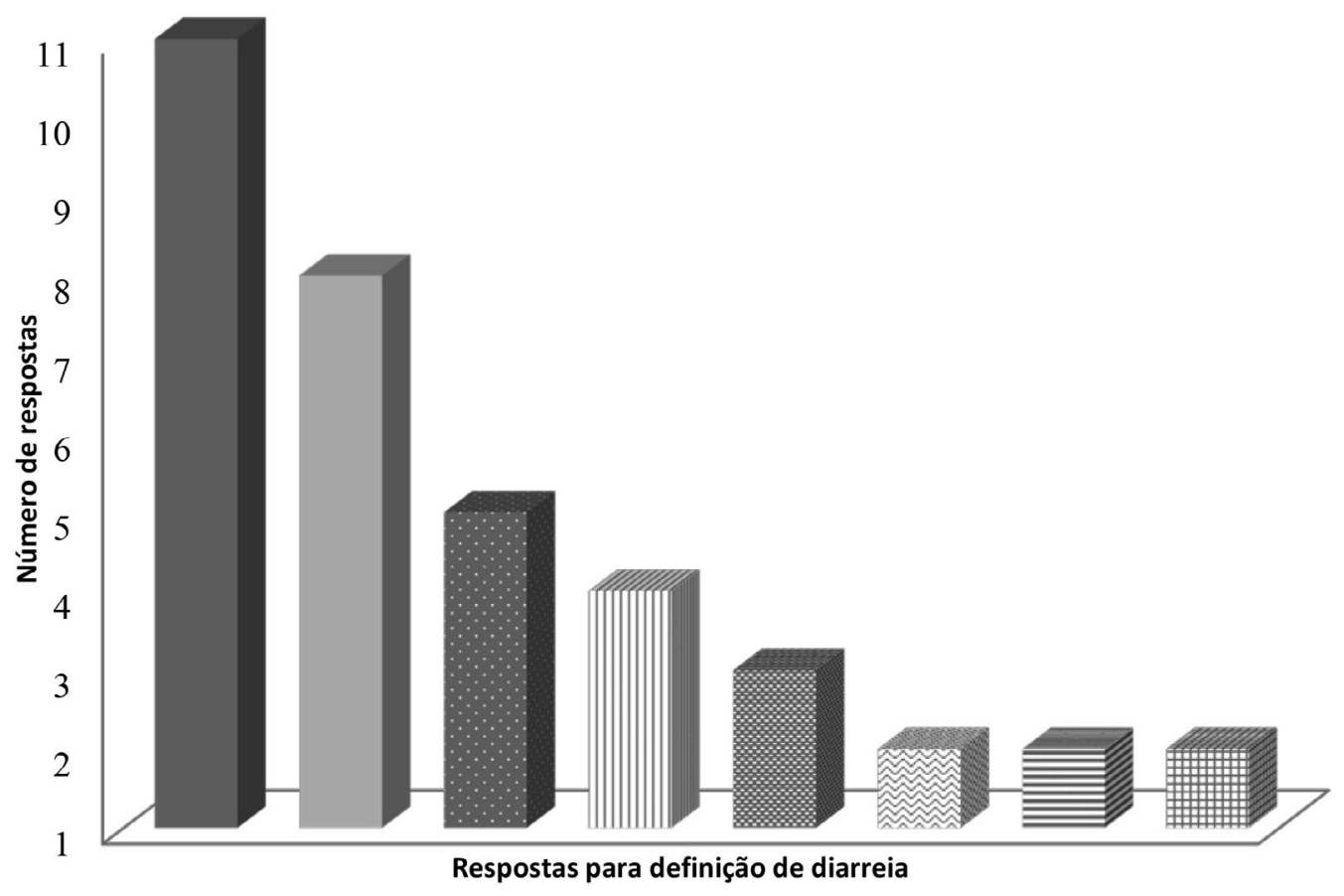

— 3 ou mais episódios de evacuações líquidas ou semi líquidas/dia Vários episódios de evacuações líquidas/dia

- Outras

I| Aumento na quantidade e alteração na consistência das fezes

臣 4 ou mais episódios de evacuações líquidas ou semi líquidas/dia

§ 5 ou mais episódios de evacuações líquidas ou semi-líquidas/dia

= Anormalidade/algo errado no intestino

\#Omissos

Outras respostas obtidas pelas questões discursivas estão demonstradas na tabela 2. A respeito da atitude/ conduta ao se deparar com um paciente com diarreia, a resposta mais frequente (19; 51,3\%) foi "comunicação à equipe multiprofissional". Em relação às causas da diarreia, as respostas mais comuns foram a "presença de infecção", e a "dieta", ambas com 19 respostas (51,3\%).

Ainda considerando as questões discursivas, em relação aos motivos pelos quais a dieta possa causar diarreia, em 15 (40,5\%) questionários foi citado o "aporte calórico e volume inadequado (em excesso)". Em segundo lugar, a "composição da dieta" foi citada em 14 (37,8\%) questionários Os itens da composição da dieta associados à diarreia foram: módulo de proteína, açúcar, fibras, lipídios e ingredientes laxantes. Na maioria dos questionários (12; 32,4\%), foi citado que "adequar a dieta" é uma medida para se prevenir a interrupção da mesma em casos de diarreia, seguidos de 10 (27\%) que mencionaram que a "capacitação da equipe multiprofissional" é essencial. 
Tabela 2. Conhecimento e conduta de profissionais de saúde em relação à diarreia em pacientes recebendo nutrição enteral - Uberlândia, MG - 2017

\begin{tabular}{|c|c|c|}
\hline Respostas obtidas pelos profissionais & $\mathbf{n}$ & $\%$ \\
\hline \multicolumn{3}{|c|}{ Conduta ao se deparar com um paciente com diarreia: } \\
\hline Comunica a equipe multiprofissional & 19 & 51,3 \\
\hline Identifica a etiologia & 11 & 29,7 \\
\hline Outras $^{1}$ & 3 & 8,1 \\
\hline \multicolumn{3}{|l|}{ Causas da diarreia: } \\
\hline Infecção & 19 & 51,3 \\
\hline Dieta $^{2}$ & 19 & 51,3 \\
\hline Fatores intrínsecos ao paciente 3 & 18 & 48,6 \\
\hline Antibióticos & 13 & 35,1 \\
\hline Outras $^{4}$ & 3 & 8,1 \\
\hline \multicolumn{3}{|c|}{ Motivos pelos quais a dieta pode causar diarreia: } \\
\hline Aporte calórico/volume em excesso & 15 & 40,5 \\
\hline Composição ${ }^{5}$ & 14 & 37,8 \\
\hline Velocidade de infusão & 10 & 27,0 \\
\hline Contaminação & 9 & 24,3 \\
\hline Osmolaridade & 6 & 16,2 \\
\hline Intolerância alimentar & 7 & 18,9 \\
\hline \multicolumn{3}{|c|}{$\begin{array}{l}\text { O que deve ser feito para se prevenir a interrupção da dieta em casos de } \\
\text { diarreia: }\end{array}$} \\
\hline Adequar a dieta ${ }^{6}$ & 12 & 32,4 \\
\hline Capacitar a equipe multiprofissional & 10 & 27,0 \\
\hline Descobrir a etiologia & 5 & 13,5 \\
\hline Outras ${ }^{7}$ & 5 & 13,5 \\
\hline Criar protocolo & 4 & 10,8 \\
\hline
\end{tabular}

1Inclui: Otimiza hidratação e higienização das mãos; analisa características das fezes; reduz velocidade de infusão de dieta; higieniza o paciente. ${ }^{2}$ Inclui: dieta com aporte calórico e/ou proteico não adaptados (em excesso); dieta contaminada; dieta em temperatura e tempo de infusão inadequados. ${ }^{3}$ Inclui: desnutrição; doenças do trato gastrointestinal; disabsorção intestinal; motilidade intestinal; intolerâncias ou alergias; fatores psicológicos. ${ }^{4}$ Inclui: Interações medicamentosas; medicamentos, exceto antibióticos. ${ }^{5}$ Inclui: módulo de proteína; açúcar; fibras; lipídios e ingredientes laxantes. ${ }^{6}$ Inclui: substituir dieta; reduzir volume e velocidade de infusão. ${ }^{7}$ Inclui: Infundir medicação; infundir alimento obstipante (suco de caju).

As respostas obtidas pelas questões de múltipla escolha estão demonstradas na tabela 3. A maioria dos profissionais, (20; 54\%), não recebeu nenhum tipo de treinamento sobre diarreias, destacando-se a profissão de técnico/ auxiliar de enfermagem, onde 12 (70,6\%) não receberam. Dos profissionais que já receberam algum tipo de treinamento, a maioria $(10 ; 27 \%)$, foi somente em faculdade ou curso técnico. 
Tabela 3. Respostas obtidas nas questões de múltipla escolha sobre o conhecimento e conduta de profissionais de saúde em relação à diarreia em pacientes recebendo nutrição enteral - Uberlândia, MG - 2017

\begin{tabular}{|c|c|c|}
\hline Respostas obtidas pelos profissionais & $\mathbf{n}$ & $(\%)$ \\
\hline \multicolumn{3}{|l|}{ Recebeu algum tipo de treinamento sobre diarreias: } \\
\hline Sim, na faculdade/curso técnico & 10 & 27,0 \\
\hline Sim, em serviço em UTI & 2 & 5,4 \\
\hline Sim, na faculdade/curso técnico e em serviço em UTI & 5 & 13,5 \\
\hline Não & 20 & 54,0 \\
\hline \multicolumn{3}{|l|}{ Registro de casos de diarreia: } \\
\hline Não registra & 1 & 2,7 \\
\hline Comunica verbalmente & 4 & 10,8 \\
\hline Registra sem maiores informações & 6 & 16,2 \\
\hline Registra detalhadamente & 25 & 67,6 \\
\hline \multicolumn{3}{|c|}{ Vê dietas interrompidas por causa de diarreia com qual frequência: } \\
\hline Pouca & 11 & 29,7 \\
\hline Razoável & 9 & 24,3 \\
\hline Muita & 16 & 43,2 \\
\hline \multicolumn{3}{|l|}{ Conhece algum protocolo sobre diarreia: } \\
\hline Sim & 2 & 5,4 \\
\hline Não & 33 & 89,1 \\
\hline \multicolumn{3}{|l|}{ Considera importante a criação de um protocolo: } \\
\hline Sim & 33 & 89,1 \\
\hline Não & 2 & 5,4 \\
\hline
\end{tabular}

Quando se deparam com um paciente com diarreia na prática clínica, a maioria dos profissionais (25; 67,6\%) faz registro detalhado dos casos em relatórios próprios ou prontuários. Nas UTIs estudadas não existe protocolo a ser seguido em casos de diarreia, e a maioria dos profissionais $(33,89,1 \%)$ nunca teve contato com algum, considerando importante sua implementação.

\section{Discussão}

Foram observadas divergências e falta de padronização no conhecimento e nas condutas dos profissionais de saúde que participaram deste estudo. Quando os profissionais de saúde foram questionados sobre a definição de diarreia, observaram-se respostas heterogêneas, o que pode ser refletido pela formação do profissional, tam-

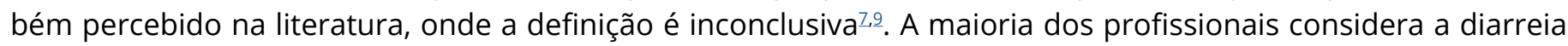
como a presença de 3 ou mais evacuações líquidas ou semilíquidas/dia, definição dada pela Organização Mundial da Saúde (2005) $)^{10}$. Um fato preocupante, que reflete no tratamento do paciente, é que não há um consenso entre profissionais de um mesmo serviço, ou seja, não há padronização no conceito do tema abordado.

Quanto às causas da diarreia, a maioria dos participantes cita a "dieta" como um dos fatores causais. Assim como visto em outro estudo ${ }^{10}$, a dieta como fator causal foi citada, em maior parte, pelos auxiliares/técnicos em enfermagem. Essa resposta pode ser justificada pela falta de treinamento pela maioria desses profissionais.

Juntamente com a "dieta", o item "infecção" foi apontado como uma das causas mais citadas para a ocorrência de diarreia, também um dos itens mais citados no estudo de Lordani et al. ${ }^{11}$. A infecção como fator causal da diarreia foi encontrada, em sua maioria, nos questionários respondidos pelos profissionais médicos $(87,5 \%)$, incluindo infeç̧ão por Clostridium difficile - bactéria anaeróbica gram-positiva responsável pela maior parte de casos de diarreia associada ao uso de antibióticos ${ }^{13,14}$. No estudo de Lordani et al.11, a "infecção gastrointestinal" foi o terceiro fator causal mais citado entre os profissionais de saúde. 
Os "antibióticos" foram citados por $35,1 \%$ dos profissionais como sendo fator causal da diarreia. O uso desses medicamentos está associado a um distúrbio na microbiota bacteriana normal do cólon, o que pode promover a colonização por Clostridium difficile. A diarreia acomete cerca de 5 a 30\% dos pacientes em uso de antibióticos, podendo logo após a primeira dose ou até dois meses após o final do tratamento ${ }^{15}$. Em uma revisão bibliográfica realizada em 2018, foi visto que em mais de $30 \%$ dos estudos foi constatada a diarreia em UTI, sendo que a maioria foi relacionada ao uso de antibióticos $\frac{16}{}$.

Em relação aos "fatores intrínsecos do paciente", foram encontrados aspectos como "desnutrição; doenças do trato gastrointestinal; disabsorção intestinal; motilidade intestinal; intolerâncias ou alergias e fatores psicológicos", citados pela maioria dos profissionais enfermeiros e nutricionistas. Sabe-se que o paciente, além de, possivelmente, apresentar doenças gastrointestinais de base, podem ter suas funções fisiológicas alteradas na doença crítica 2 . Diferentes complicações gastrointestinais, como diminuição de ruídos intestinais, retardo no esvaziamento gástrico e diarreia podem ocorrer em até $50 \%$ dos pacientes em ventilação mecânica e a intolerância à nutrição enteral em até $46 \%$ dos pacientes que fazem o uso de vasopressores ${ }^{2}$.

Em relação aos motivos pelos quais a dieta possa causar diarreia, o "aporte calórico/volume em excesso", a "composição", a "velocidade de infusão" e a "contaminação" foram os itens mais citados. No item "dieta com aporte calórico/volume em excesso", citado pela maioria dos auxiliares/técnicos em enfermagem, observamos um conhecimento errôneo, visto que menos da metade dos pacientes internados em UTI recebem o total de suas necessidades nutricionais ${ }^{4}$, sendo mais comum ocorrer um déficit na oferta, visto que comumente as infusões de dietas são interrompidas (em aproximadamente $85 \%$ dos pacientes e em média de 8 a $20 \%$ do tempo de infusão) $)^{4}$. Além disso, é importante destacar que o cálculo das necessidades nutricionais e a prescrição dietética são realizados por profissionais nutricionistas, responsáveis e capacitados para essas funções.

A "composição da dieta" foi citada pela metade dos enfermeiros e por alguns dos auxiliares/técnicos em enfermagem consideraram o módulo de proteína, açúcar, fibras, lipídios e ingredientes laxantes.
Nas UTI's estudadas, todas as dietas são industrializadas e as poliméricas mais utilizadas são líquidas e a oligomérica, em pó. Uma das dietas poliméricas é normocalórica $(1,2 \mathrm{kcal} / \mathrm{mL})$, com $21 \%$ de proteínas, $45 \%$ de carboidratos e $34 \%$ de gorduras e sem fibras e a outra, hipercalórica $(1,5 \mathrm{kcal} / \mathrm{mL})$, com $17 \%$ de proteínas, $41 \%$ de carboidratos e $42 \%$ de gorduras e com fibras solúveis (maioria) e insolúveis. Nenhuma delas contém sacarose (açúcar). A dieta oligomérica contém $16 \%$ de proteínas, $49 \%$ de carboidratos (sendo $25 \%$ de sacarose) e $35 \%$ de gorduras e não contém fibras.

De acordo com as recomendações realizadas pelas Dietary Reference Intakes ${ }^{17}$ de macronutrientes para adultos (carboidratos de 45 a 65\%, proteínas de 10 a $35 \%$ e gorduras de 20 a 35\%) e de fibras (38 g/dia para homens adultos com até 50 anos e $25 \mathrm{~g} /$ dia para mulheres adultas com até 50 anos), observa-se que as dietas mais utilizadas não ultrapassam as recomendações, exceto a dieta polimérica hipercalórica que apresenta quantidade elevada de gorduras (42\%). Um ponto positivo desta dieta é a presença de um mix de fibras ( $8 \mathrm{~g} /$ litro). Sugere-se que pacientes com diarreia persistente podem se beneficiar com o uso de dietas contendo um mix de fibras. Porém, a suplementação de fibras solúveis parece ser mais benéfica para a redução de diarreia nos pacientes em estado crítico 3 . Um aditivo de fibras solúveis (10-20 g/24 horas) é recomendado para os pacientes de UTI que apresentarem evidência de diarreia como forma de manter a microbiota comensal e promover a saúde intestinal. Devendo considerar que o uso de fibras é contraindicado em pacientes com risco de isquemia intestinal ou dismotilidade grave ${ }^{3}$, fato que deve ser considerado pela equipe de nutricionistas e médicos que participam da prescrição de dieta enteral.

Além da composição da própria dieta, foi citado o "módulo de proteína" como fator que pode causar a diarreia. O módulo é acrescentado na maioria das dietas enterais dos pacientes internados nas UTI's estudadas para se atingir a recomendação proteica. Na prática clínica, existe uma cultura entre os profissionais de que esse módulo possa aumentar o risco de o paciente desenvolver diarreia ou agravá-la, se já existir. O módulo utilizado é composto por 100\% de proteína do soro do leite e não contém adição de carboidratos e gorduras. Não foram encontrados na literatura informações a respeito da oferta de proteínas na etiologia da diarreia. 
A "velocidade de infusão" foi citada pela maioria dos nutricionistas. Sabe-se que esse é um fator que deve ser observado na ocorrência de diarreia. Porém, na prática clínica das UTI's estudadas, todas as dietas são ofertadas por bomba de infusão com tempo de aproximadamente 15 horas/dia. Contudo, acredita-se que esse não é um fator causal, visto que, a infusão lenta da dieta enteral pode contribuir para diminuir o problema de intolerância a uma dieta hiperosmolar, por exemplo $2,$. .

Em relação à "contaminação" e "dieta em temperatura inadequada" (citada em "causas da diarreia"), destaca-se que é função do enfermeiro a conservação após o recebimento da dieta enteral e sua administração ${ }^{18}$. Assim, se os próprios profissionais dessa categoria profissional citam essas questões, salienta-se a importância de um treinamento a respeito da correta conservação e administração das dietas enterais.

A maioria dos profissionais médicos citou como motivo a "osmolaridade", também citada pela maioria dos nutricionistas e não citada por nenhum profissional da enfermagem. A osmolaridade pode causar intolerância gastrointestinal devido efeitos osmóticos, sendo que, dietas administradas no estômago podem conter osmolaridade mais elevada enquanto dietas administradas no intestino devem ser isoosmolares ${ }^{2}$. Das dietas mais utilizadas nas UTI's estudadas, a polimérica hipercalórica apresenta osmolaridade de $320 \mathrm{mOsm} / \mathrm{kg}$ de água (isotônica), a polimérica normocalórica $391 \mathrm{mOsm} / \mathrm{kg}$ de água (levemente hipertônica) e a oligomérica, $375 \mathrm{mOsm} / \mathrm{kg}$ de água (levemente hipertônica). Ou seja, nenhuma das dietas mais utilizadas apresenta osmolaridade elevada (> $550 \mathrm{mOsm} / \mathrm{kg}$ de água). Além das dietas, é importante salientar que algumas medicações líquidas são hiperosmolares, o que também pode ser um fator agravante $\frac{15}{}$.

Sabe-se que fatores relacionados diretamente à nutrição enteral podem estar envolvidos na etiologia da diarreia, como a quantidade de fibras e gorduras, densidade calórica, osmolaridade, temperatura, velocidade de infusão e contaminação da dieta2,7,9,16. Sendo assim, vê-se que as respostas obtidas pelos profissionais estão condizentes com a literatura. Porém, assim como visto no estudo de Lordani et al.111 não há um consenso entre os profissionais.
Dentre as condutas a serem realizadas em casos de paciente com diarreia, a principal é a verificação dos possíveis fatores desencadeantes, ao invés de se suspender a infusão da dieta enteral ${ }^{2} \underline{1}$. No presente estudo, pode-se observar a conduta de se identificar a etiologia como a principal entre médicos e nutricionistas. Já para os profissionais técnicos e auxiliares em enfermagem e enfermeiros, a principal conduta ao se deparar com um paciente com diarreia é a comunicação à equipe multiprofissional. Em adição, a maioria dos profissionais em enfermagem faz registro detalhado dos casos de diarreia, em concordância com os resultados encontrados em outro estudo ${ }^{11}$.

Embora os profissionais que presenciem com muita frequência a interrupção de dieta em casos de diarreia, apenas um deles citou esta conduta, que, na opinião dele deve ser realizada somente depois da tentativa fracassada de se identificar a etiologia da diarreia e de se alterar o tipo de dieta. Sendo assim, fica interrogado se houve omissão dessa resposta pelos profissionais quando questionados sobre suas atitudes/condutas.

Em uma revisão bibliográfica realizada por Pinheiro et al. $\frac{19}{}$, foi visto que em $60 \%$ dos estudos analisados, a intolerância gastrointestinal foi o motivo para interrupção de dieta enteral em pacientes internados em UTI e em 20\%, a diarreia. No estudo de Quaresma et al. 20 a interferência na dieta enteral em casos de diarreia foi vista de forma mais significativa, onde a maioria dos médicos intensivistas estudados têm como conduta reduzir a taxa de infusão da dieta enteral e $13 \%$ deles interrompem a dieta por 24 horas.

No estudo de Majid et al. ${ }^{21}$, as principais condutas de enfermeiros e nutricionistas são "monitorar as evacuações" e "enviar amostra fecal para análises microbiológicas". No presente estudo, 3 profissionais médicos citaram como conduta a solicitação de exames das fezes, como coprocultura, exame parasitológico e pesquisa de toxinas A e B para infecção por Clostridium difficile.

A "adequação da dieta" e a "capacitação da equipe multiprofissional" foram os itens mais citados como sendo soluções para se prevenir a interrupção da dieta em casos de diarreia. Os profissionais técnicos/auxiliares em enfermagem compuseram a maioria dos que acreditam que se deva adequar à dieta nesses casos. 
Sabe-se que o manejo da dieta pode aliviar a diarreia na alimentação enteral ${ }^{7}$, porém, a principal conduta a ser tomada deve ser a identificação da etiologia para se manejar o fator causal.

Para a maioria dos profissionais médicos e nutricionistas no presente estudo, a capacitação da equipe multiprofissional foi a solução citada como forma de se prevenir a interrupção da dieta em casos de diarreia. Esse é um fator essencial, visto que 20 profissionais (54\%) não receberam nenhum tipo de treinamento sobre o tema.

Após treinamento da equipe de enfermagem, há redução nos diagnósticos equivocados de diarreia em pacientes recebendo nutrição enteral ${ }^{22}$. Em um estudo realizado por Barbosa et al. ${ }^{23}$ foi visto que a equipe de enfermeiros de uma UTI possui, de forma geral, conhecimento satisfatório a respeito da terapia nutricional, possivelmente justificada por treinamentos realizados pela instituição. $\mathrm{O}$ manejo adequado da diarreia, assim como de outras complicações gastrointestinais em pacientes recebendo dieta enteral, depende do conhecimento da equipe multiprofissional. Treinamentos constantes e instrumentos que facilitem identificar e manejar as complicações favorecem redução de desfechos negativos ao paciente ${ }^{24,25}$.

No presente estudo, a maioria dos profissionais não conhece nenhum tipo de protocolo a ser seguido, bem como considera importante a implementação de um. É importante enfatizar que protocolos bem definidos devem ser implementados para o tratamento da diarreia ${ }^{15}$.

A limitação do estudo foi o fato de que o questionário foi entregue por uma nutricionista, o que pode interferir nas respostas dos profissionais.

\section{Conclusão}

O presente estudo evidenciou que há divergências entre os profissionais de saúde, tanto em seus conhecimentos quanto nas condutas frente ao paciente com diarreia na UTI. O conhecimento sobre a definição de diarreia e as causas da mesma, influencia diretamente na conduta a ser tomada, e foi visto que há percepções diferentes sobre esses fatores, o que pode influenciar diretamente no cuidado ao paciente, podendo trazer prejuízos no cuidado com o mesmo.
Visto que grande parte dos profissionais não recebeu treinamento sobre diarreias, promover capacitação da equipe multiprofissional é essencial. Além disso, a criação de um protocolo seria ponto chave para padronização e consenso entre as condutas dos profissionais, a fim de se obter um manejo adequado nas UTI's estudadas.

\section{Contribuições dos autores}

Siqueira BSS participou da concepção e desenho do estudo, coleta, análise e interpretação dos dados e elaboração do artigo. Melo FG participou da concepção e desenho do estudo, interpretação dos dados, revisão do artigo e aprovação da versão final do artigo científico.

\section{Conflitos de interesses}

Nenhum conflito financeiro, legal ou político envolvendo terceiros (governo, empresas e fundações privadas, etc.) foi declarado para nenhum aspecto do trabalho submetido (incluindo, mas não se limitando a subvenções e financiamentos, participação em conselho consultivo, desenho de estudo, preparação de manuscrito, análise estatística, etc.).

\section{Referências}

1. Portaria n. 2338, de 3 de outubro de 2011 (Brasil). Estabelece diretrizes e cria mecanismos para a implantação do componente Sala de Estabilização (SE) da Rede de Atenção às Urgências. [Internet]. Diário Oficial da União; 2011. Disponível em: http://bvsms.saude.gov.br/bvs/saudelegis/gm/2011/ prt2338_03_10_2011.html

2. Toledo D, Castro M. Terapia nutricional em UTI. 1a ed. Rio de Janeiro: Rubio; 2015.

3. McClave SA, Taylor BE, Martindale RG, Warren MM, Johnson $\mathrm{DR}$, Braunschweig $\mathrm{C}$, et al. Guidelines for the provision and assessment of nutrition support therapy in the adult critically III patient: Society of Critical Care Medicine (SCCM) and American Society for Parenteral and Enteral Nutrition (A.S.P.E.N.). JPEN J Parenter Enteral Nutr. 2017;40(2):159-211. https://doi. org/10.1177/0148607115621863
4. Chung CK, Whitney R, Thompson CM, Pham TN, Maier RV O'Keefe GE. Experience with an enteral-based nutritional support regimen in critically ill trauma patients. J Am Coll Surg. 2013;217(6):1108-17. https://dx.doi.org/10.1016\%2Fj. jamcollsurg.2013.08.006 
5. Lobato TAA, Garla PC. Monitoramento da terapia nutricional enteral em doentes críticos no Brasil: uma revisão. BRASPEN J. 2020;35(2):166-70. https://dx.doi.org/10.37111/ braspenj.2020352010

6. Passier RHA, Davies AR, Ridley E, McClure J, Murphy D, Scheinkestel CD. Periprocedural cessation of nutrition in the intensive care unit: opportunities for improvement. Intensive Care Med. 2013;39(7):1221-6. https://doi.org/10.1007/s00134-0132934-8

7. Chang SJ, Huang HH. Diarrhea in enterally fed patients: blame the diet? Curr Opin Clin Nutr Metab Care. 2013;16(5):588-94. https://doi.org/10.1097/mco.0b013e328363bcaf

8. Rushdi TA, Pichard C, Khater YH. Control of diarrhea by fiberenriched diet in ICU patients on enteral nutrition: a prospective randomized controlled trial. Clin Nutr. 2004;23(6):1344-52. https:// doi.org/10.1016/j.clnu.2004.04.008

9. Ferrie S, East V. Managing diarrhoea in intensive care. Aust Crit Care. 2007;20(1):7-13. https://doi.org/10.1016/j.aucc.2006.10.001

10. World Health Organization. The treatment of diarrhoea. A manual for physicians and other senior health workers [Internet]. Geneva: World Health Organization; 2005. Disponível em: http:// whqlibdoc.who.int/publications/2005/9241593180.pdf

11. Lordani CRF, Eckerti RG, Tozzeto AG, Lordani TVA, Duarte PAD. Conhecimento de profissionais de unidade de terapia intensiva sobre diarreia. Rev Bras Ter Intensiva. 2014;26(3):299-304. https:// doi.org/10.5935/0103-507X.20140042

12. Telles JLH, Boton CRM, Mariano MLL, Paula MAB. Nutrição enteral: complicações gastrointestinais em pacientes de uma unidade de terapia intensiva. Revista Recien. 2015;5(13):5-11. https://doi.org/10.24276/rrecien2358-3088.2015.5.13.5-11

13. Freedberg DE, Salmasian H, Cohen B, Abrams JA, Larson EL. Receipt of antibiotics in hospitalized patients and risk for Clostridium difficile infection in subsequent patients who occupy the same bed. JAMA Intern Med. 2016;176(12):1801-8. https://doi. org/10.1001/jamainternmed.2016.6193

14. Geoghegan O, Eades C, Moore LSP, Gilchrist M. Clostridium difficile: diagnosis and treatment update. Clinical Pharmacist [Internet]. 2017;9(2):1-15. Disponível em: https://pharmaceuticaljournal.com/article/ld/clostridium-difficile-diagnosis-andtreatment-update

15. AlKhawaja S. Diarrhea in Critically III Patient. EC Nutrition 9.2 [Internet]. 2017;105-15. Disponível em: https://www.ecronicon. com/ecnu/pdf/ECNU-09-00299.pdf
16. Campos MF, Brito LMZ, Carvalho ND, Santos SL, Alencar MAVSD, Coelho CH. Nutrição enteral e a ocorrência de diarreia no paciente crítico. International Journal of Nutrology. 2018;11(1):24327. https://doi.org/10.1055/s-0038-1674845

17. Institute of Medicine. Dietary Reference Intakes for Energy, Carbohydrate, Fiber, Fat, Fatty Acids, Cholesterol, Protein, and Amino Acids. Washington, DC: The National Academies Press; 2005. https://doi.org/10.17226/10490

18. Resolução RDC $n^{\circ}$ 63, 6 de Julho de 2000 (Brasil). Aprova o Regulamento Técnico para fixar os requisitos mínimos exigidos para a Terapia de Nutrição Enteral. [Internet] Diário Oficial da União. Disponível em: http://bvsms.saude.gov.br/bvs/saudelegis/ anvisa/2000/rdc0063_06_07_2000.html

19. Pinheiro DS, Santo FHE, Ribeiro WA, Fassarella BPA. Fatores de interrupção de nutrição enteral em unidades de terapia intensiva adulto: revisão integrativa. RSD. 2020;9(9): e985998188. http:// dx.doi.org/10.33448/rsd-v9i9.8188

20. Quaresma EN, Villacorta DBV, Silva RA, Veríssimo AOL, Caldato MCF. Análise da compreensão a respeito da terapia nutricional em Unidades de Terapia Intensiva de um hospital escola. REAS. 2019;11(15):e1338. https://doi.org/10.25248/reas.e1388.2019

21. Majid HA, Emery PW, Whelan K. Definitions, attitudes and management practices in relation to diarrhea during enteral nutrition: A survey of patients, nurses, and dietitians. Nutr Clin Pract. 2012;27(2):252-60. https://doi. org/10.1177/0884533611431986

22. Kumbier M, Costa C, Barreto AL, Abreu AR, Gonzáles D, Spolidoro JV. Análise dos registros de evacuações pela equipe de enfermagem em pacientes com nutrição enteral (NE): redução de registro de diarreias após treinamento. Rev Bras Nutr Clín [Internet]. 2009;24(3):155-8. Disponível em: http://pesquisa. bvsalud.org/bvsvs/resource/pt/lil-550

23. Barbosa JAG, Carlos CM, Costa RF, Simino GPR. Conhecimento de enfermeiros acerca da terapia nutricional. Rev Enferm Contemp. 2020;9(1):33-40. http://dx.doi.org/10.17267/23173378rec.v9i1.2543

24. Sampaio IR, Ferrari TKV, Toso TP, Duarte LM, Luzzi LC, Souza VV, et al. Analysis of the adequacy of quality indicators in enteral nutrition therapy in an intensive care unit. RSD. 2019;8(12):e468121941. https://doi.org/10.33448/rsd-v8i12.1941

25. Gomes KOAC, Mesquita DT, Sousa LS, Castro RA, Portella EMM, Sousa US, et al. Intercorrências nutricionais em pacientes críticos: uma percepção multiprofissional. REAS. 2018;10(3):162833. https://doi.org/10.25248/REAS205_2018 